\title{
TREES, LATTICES, ORDER, AND BETWEENNESS ${ }^{1}$
}

\author{
MARLOW SHOLANDER
}

In this paper we consider postulates expressed in terms of "segments," "medians," and "betweenness." Characterizations are obtained for trees, lattices, and partially ordered sets. In general a characterization is given by a system of three postulates. These systems fall in pairs; systems of a pair have two postulates in common. An algebra which has both lattices and trees as special cases is given in the final section.

1. Segments. Consider a set $S$ of elements $a, b, c, \cdots$ such that to each pair $a, b$, of elements in $S$ there corresponds a unique subset of $S$ denoted by $(a, b)$ and called the segment from $a$ to $b$. By assumption, these segments have as properties:

(S) To each set of three elements $a, b$, and $c$, there corresponds an element $d$ such that $(a, b) \cap(b, c)=(b, d)$.

(T) $(a, b) \subset(a, c)$ implies $(a, b) \cap(b, c)=\{b\}$.

The segments of Duthie [1] $]^{2}$ are segments in this sense (see $\S 3$ ). Paths in a tree are also segments in this sense (see \$2).

Setting $a=b=c$ in (T) we have

$$
\{a\}=(a, a) .
$$

From this, and from (T) with $b=c$, we have

$$
\begin{aligned}
& b \in(a, b) . \\
& a \in(a, b) .
\end{aligned}
$$

Proof. From (S), we may choose $d$ so that $(a, a) \cap(a, b)=(a, d)$. By (1.1) and (1.2), $d \in(a, d) \subset(a, a)=\{a\}$. Hence $d=a$ and $a \in(a, d)$ $C(a, b)$.

$$
(a, b)=(b, a) \text {. }
$$

Proof. From (S), we may choose $d$ so that $(a, b) \cap(b, a)=(b, d)$. By (1.2) and (1.3), $a \in(b, d)$. By (T), $(b, d) \cap(d, a)=\{d\}$. Hence $a \in\{d\}, a=d$, and $(b, a)=(b, d) \subset(a, b)$. By symmetry, $(a, b) \subset(b, a)$.

$$
b \in(a, c) \text { if and only if }(a, b) \subset(a, c) \text {. }
$$

Received by the editors June 12, 1950 and, in revised form, September 20, 1951.

1 This paper was written while the author was under contract to the Office of Naval Research.

${ }^{2}$ Numbers in brackets refer to the bibliography at the end of the paper. 
Proof. The sufficiency proof is trivial. To prove necessity choose $d$ so that $(b, a) \cap(a, c)=(a, d)$. Clearly $b \in(a, d)$. From (1.4) and (T), $(a, d) \cap(d, b)=\{d\}$. Hence $b \in\{d\}, b=d$, and $(a, b)=(a, d) \subset(a, c)$.

From (1.5) and (T) we have

$$
\begin{aligned}
& b \in(a, c) \text { implies }(a, b) \cap(b, c)=\{b\} . \\
& b \in(a, c) \text { and } c \in(a, b) \text { imply } b=c .
\end{aligned}
$$

Thus if $(a, b)=(a, c)$ we have $b=c$.

Proof. By (1.5), $(a, b) \subset(a, c) \subset(a, b)$. Hence these segments are equal. From (1.4) and (1.6), $\{b\}=(a, b) \cap(b, c)=(a, c) \cap(c, b)=\{c\}$.

As a corollary of (1.7) we have

The element $d$ of $(\mathrm{S})$ is unique.

$$
b \in(a, c) \text { if and only if }(a, b) \cup(b, c) \subset(a, c) \text {. }
$$

Proof. The condition is necessary by (1.4) and (1.5). It is sufficient by (1.2) and (1.3).

$$
(a, b) \cap(b, c)=(b, d) \text { implies }(a, d) \cap(d, c)=\{d\} .
$$

Proof. We note $d \in(a, b)$ and $d \in(b, c)$. From (1.9), $(a, b) \supset(a, d)$ $\cup(d, b)$ and $(b, c) \supset(b, d) \cup(d, c)$. Taking set intersections we obtain $(b, d) \supset(b, d) \cup[(a, d) \cap(d, c)]$. From this and from (1.6), $(a, d)$ $\bigcap(d, c)=(a, d) \cap(d, c) \cap(b, d)=\{d\} \cap(d, c)=\{d\}$.

2. Tree segments. The word tree is probably most often used in mathematics to denote a finite connected linear graph which contains no cycles [2, p. 47]. However a connected acyclic union of closed Jordan arcs is sometimes called a tree and in Lattice theory [3, p. 47] we find the word used in still a third sense. Seeking characteristics common to the several types of objects known as trees we arrive at the definition below. (Birkhoff's trees can be imbedded in our trees. Trees in our sense which are finite are trees in König's sense.)

A tree is defined as a set of elements which satisfies (S), (T), and:

$\left(\mathrm{U}_{1}\right)(a, b) \cap(b, c)=\{b\}$ implies $(a, b) \cup(b, c)=(a, c)$.

From this definition, (1.9), and (1.6) we have

(2.1) $b \in(a, c),(a, b) \cap(b, c)=\{b\}$, and $(a, b) \cup(b, c)=(a, c)$ are equivalent conditions.

(2.2) $(a, b) \cap(b, c)=(b, d)$ if and only if $\{d\}=(a, b) \cap(b, c) \cap(c, a)$. Hence the last term always represents a set containing a single point.

Proof. If $(a, b) \cap(b, c)=(b, d)$, we have by (1.10) that $(a, d)$ $\cap(d, c)=\{d\}$. Using (2.1) and the distributive law, we have $(a, b)$ 
$\cap(b, c) \cap(c, a)=(b, d) \cap(c, a)=(b, d) \cap[(a, d) \cup(d, c)]=\{d\} \cup\{d\}$ $=\{d\}$. The sufficiency of the condition follows from (S) and the necessity of the condition.

Definition. The unique element given by $(a, b) \cap(b, c) \cap(c, a)$ is called the median of $a, b$, and $c$ and is denoted by $(a, b, c)$. Properties of this ternary operation are derived in $\$ 5$.

For all $a, b$, and $c,(a, b) \subset(a, c) \cup(c, b)$.

Proof. Let $d=(a, b, c)$. From $(2.1),(a, b)=(a, d) \cup(d, b)$. From (1.5), $(a, d) \subset(a, c)$ and $(d, b) \subset(c, b)$.

Definition. We say $b$ is between $a$ and $c$ and write $a b c$ if and only if $b \in(a, c)$.

As a consequence of (1.1) we have

$a b a$ if and only if $a=b$.

As a consequence of (2.2) we have

(2.5) To elements $a, b$, and $c$ there corresponds a unique element $d$ such that $a d b, b d c$, and $c d a$.

(2.6) If we have both $a b c$ and $b d e$, then we have either $c b d$ or $e b a$, perhaps both.

Proof. Let $b \in(a, c)$ and $d \in(b, e)$. By (2.3), if $b \notin(a, e)$ then $b \in(e, c)$. Again, if $b \in(c, d)$ then $b \in(d, e)$. But from (1.7) we have in this case the contradiction $b=d \in(c, d)$.

3. Lattice segments. Consider a set $S$ satisfying (S), (T), and:

$\left(\mathrm{U}_{2}\right)$ There are elements 0 and $I$ in $S$ such that if $(0, r) \cap(0, s)$ $C(0, a) \cap(0, b)$ and $(I, r) \cap(I, s) \subset(I, a) \cap(I, b)$, then $(a, b) \subset(r, s)$.

$$
r \in(0, a) \text { if and only if } a \in(r, I) .
$$

Proof. Let $b=s=I$ in $\left(\mathrm{U}_{2}\right)$ to prove necessity. The proof of sufficiency is dual.

Letting $r=0$ in (3.1), we have

$$
\text { For all } a, a \in(0, I) \text {. }
$$

(3.3) If $(0, a) \cap(0, b)=(0, r)$ and $(I, a) \cap(I, b)=(I, s)$ then $(a, b)=(r, s)$.

Proof. From $s \in(I, a) \cap(I, b)$ and from (3.1) we have that $a$ and $b \in(0, s)$. Hence $(0, r)=(0, a) \cap(0, b) \subset(0, s)$. By (3.1), $(I, s) \subset(I, r)$. A double application of $\left(\mathrm{U}_{2}\right)$ gives $(a, b) \subset(r, s)$ and $(r, s) \subset(a, b)$.

Definition. We write $r=a b$ if and only if $(0, r)=(0, a) \cap(0, b)$. 
We write $s=a+b$ if and only if $(I, s)=(I, a) \cap(I, b)$.

$S$ is a lattice with bounds 0 and $I$.

Proof. The commutative, associative, and idempotent laws are easily derived. Properties of 0 and $I$ follow easily from (3.2). In proving an absorption law, we let $a+b=c$ and $a c=d$. From $(I, c)=(I, a)$ $\cap(I, b)$ and (3.1), we have $a$ in $(0, c)$. Hence $(0, a)=(0, a) \cap(0, c)$ $=(0, d)$. By (1.7), $a=d$. The other absorption law follows dually.

$$
x \in(a, b) \text { if and only if } a b \leqq x \leqq a+b .
$$

Proof. By (3.3), $x \in(r, s)$ where $r=a b$ and $s=a+b$. As in the proof of (3.3), $(0, r) \subset(0, s)$. By (1.5), $(r, s) \subset(0, s)$. By (3.1), $s \in(I, x)$. Hence $(I, s) \subset(I, x)$ and $x+s=s$. Dually, $x r=r$. To prove the inequality sufficient, we note $x r=r$ implies $(0, r) \subset(0, x)$. Similarly, $(I, s) \subset(I, x)$. Hence $(0, a) \cap(0, b) \subset(0, a) \cap(0, x)$ and $(I, a) \cap(I, b)$ $C(I, a) \cap(I, x)$. By $\left(\mathrm{U}_{2}\right),(a, x) \subset(a, b)$ and $x \in(a, b)$.

(3.6) Postulates $\mathrm{S}, \mathrm{T}$, and $\mathrm{U}_{2}$ characterize distributive lattices with 0 and $I$.

Proof. That these postulates give a distributive lattice with 0 and $I$ follows from (3.4), (3.5), (1.6), and a theorem of Duthie [1]. Conversely, if in such a distributive lattice we define $(a, b)$ as the set of all $x$ such that $a b \leqq x \leqq a+b$, the three postulates are easily derived.

4. Betweenness. We now consider a set $S$ of elements $a, b, c, \cdots$ in which, for each ordered set of three elements $a, b$, and $c$, there holds or fails to hold a relation denoted by $a b c$ and read " $b$ is between $a$ and $c . "$ This relation satisfies the following postulates:

(B) $a b a \leftrightarrow a=b$.

(C) $a b c \cdot b d e \rightarrow(c b d$ or $e b a)$.

For the interpretation of the notation in (C) see (2.6). We proceed to derive consequences of these two postulates.

$$
a a b \rightarrow b a a .
$$

Proof. By (C), $a a b \rightarrow a a b \cdot a a b \rightarrow(b a a$ or $b a a) \rightarrow b a a$.

$$
a b c \rightarrow a a b .
$$

Proof. If $a=c, a b c \rightarrow a b a \rightarrow a=b$. But by (B) we have $a a a$, and now $a a a \rightarrow a a b$. If $a \neq c$, from $a a a \cdot a b c \rightarrow(a a b$ or $c a a)$, we have either $a a b$ or $c a a \cdot a b c \rightarrow(a a b$ or $c a c) \rightarrow a a b$.

$$
a b c \rightarrow c b a .
$$


Proof. If $a=b$, this follows from (4.1). If $a \neq b$, we note by (4.2) and (4.1) that $a b c \rightarrow b a a$ and we then have that $a b c \cdot b a a \rightarrow(c b a$ or $a b a)$ $\rightarrow c b a$.

(4.4) Each of the relations $a a b, a b b, b b a$, and $b a a$ implies the other three.

Proof. By (4.1), $a a b \rightarrow b a a$ and $b b a \rightarrow a b b$. By (4.2), $b a a \rightarrow b b a$ and $a b b \rightarrow a a b$.

Definition. We say $a$ is comparable with $b$ and write $a b$ if and only if $a a b$ holds. It follows that $a a$ holds for all $a$ in $S$, that $a b \rightarrow b a$, and that $a b$ is equivalent to each of the betweenness relations of (4.4). It is unlikely that this notation will be confused with the product notation of $\$ 3$.

$$
a b c \rightarrow a b \cdot b c \cdot c a .
$$

Proof. By (4.2) and (4.3), $a b c \rightarrow a b$ and $a b c \rightarrow c b a \rightarrow c b$. If $a=b$, $c b \rightarrow c a$. If $a \neq b$, by (4.2) and (C), we have $a b c \rightarrow a a b \cdot a b c \rightarrow(b a b$ or $c a a)$ $\rightarrow c a a \rightarrow c a$.

Definition. We say $a_{1}, a_{2}, \cdots, a_{n}$ form a chain and denote this by $a_{1} a_{2} \cdots a_{n}$ if and only if $a_{i} a_{j} a_{k}$ holds for $1 \leqq i \leqq j \leqq k \leqq n$. We note the definition is consistent with our previous notation when $n=2,3$. Clearly, $a_{1} a_{2} \cdots a_{n}$ implies both $a_{n} a_{n-1} \cdots a_{1}$ and $a_{i} a_{i+1} \cdots a_{j}$ for $1 \leqq i \leqq j \leqq n$. Moreover when $a_{i}=a_{j}$ we have $a_{i}=a_{i+1}=\cdots=a_{j-1}=a_{j}$.

$$
a b c \cdot b c d \cdot b \neq c \rightarrow a b c d .
$$

Proof. When $b \neq c$ we have $a b c \cdot b c d \rightarrow(c b c$ or $d b a) \rightarrow a b d$. Similarly, $d c b \cdot c b a \rightarrow a c d$.

$$
a b c \cdot a c d \rightarrow a b c d .
$$

Proof. If $a=c, a b c \rightarrow a b a \rightarrow a=b$ and the implication holds. If $a \neq c, a c d \cdot c b a \rightarrow(d c b$ or $a c a) \rightarrow d c b$. If $b=c, a c d \rightarrow a b d$. Finally we have $a b d$ when $b \neq c$ by (4.6).

An easy induction proof establishes the following generalization of (4.6) and (4.7).

$$
\begin{aligned}
& a_{1} a_{2} \cdots a_{n} \cdot a_{1} a_{n} b \rightarrow a_{1} a_{2} \cdots a_{n} b, \\
& a_{1} a_{2} \cdots a_{n} \cdot a_{n-1} a_{n} b \cdot a_{n-1} \neq a_{n} \rightarrow a_{1} a_{2} \cdots a_{n} b,
\end{aligned}
$$

and for $1 \leqq i \leqq n-1$,

$$
a_{1} a_{2} \cdots a_{n} \cdot a_{i} b a_{i+1} \rightarrow a_{1} a_{2} \cdots a_{i} b a_{i+1} \cdots a_{n} .
$$

Finally, we may easily prove 


$$
\begin{aligned}
a b c \cdot a c b & \rightarrow b=c . \\
a b c \cdot b d & \rightarrow(a b d \text { or } c b d) .
\end{aligned}
$$

It may be noted that we have made no attempt to use the intensive survey of betweenness made by Pitcher and Smiley [4] because of their initial assumption that every pair of elements is comparable. (This follows from their Postulate $\beta$.) It is interesting, however, that (B) and (C) imply, in addition to Postulate $\alpha$ and (1) and (2), 40 of the 43 transitivities given in Part I of their paper. ${ }^{3}$ The three transitivities not implied are their $T_{4}, T_{7}$, and $T_{10}$. Transitivity $T_{10}$ holds, however, in order betweenness (see (6.1)), and $T_{4}$ and $T_{7}$ hold in tree betweenness (see (8.10) and (8.11)). That $T_{4}$ and $T_{7}$ do not hold in order betweenness (without the restriction $a \neq b$ ) exposes a minor error in a comment by Pitcher and Smiley [4, footnote 4].

5. Tree betweenness. Consider a set $S$ which satisfies (B), (C), and:

$\left(D_{1}\right)$ Given $a, b$, and $c$, there exists an $x$ such that $a x b \cdot b x c \cdot c x a$.

We show in (5.8) that $S$ is a tree.

First, from $\left(D_{1}\right)$ and $(4.5)$, it follows that every pair of elements is comparable.

For $a, b$ in $S, a b$ holds.

$$
a x b \cdot a y b \rightarrow(a x y b \text { or } a y x b) .
$$

Proof. From (5.1), $x y$ holds. From (4.10), $a x b \cdot x y \rightarrow(a x y$ or $b x y)$. Assume, say, axy holds. By (4.7), $a x y b$ holds.

The element $x$ in $\left(D_{1}\right)$ is unique.

Proof. Assume $y$ has the same property. From $a x b \cdot a y b$ and (5.2) we have, say, $a x y b$ and hence $a x y \cdot x y b$. By (4.7), we have $a x y c \cdot c x y b$. From $x y c, c x y$, and (4.9), $x=y$.

Definitions. The element $x$ of $\left(D_{1}\right)$ is called the median of $a, b$, and $c$ and is denoted by $(a, b, c)$. The set of all $x$ such that $a x b$ holds is called the segment from $a$ to $b$ and is denoted by $(a, b)$.

$$
(a, b) \subset(a, c) \text { implies }(a, b) \cap(b, c)=\{b\} \text {. }
$$

Proof. We have given $a x b \rightarrow a x c$. We are to show $y=b$ if and only if $a y b \cdot b y c$. Necessity of the condition follows from (5.1). Conversely, noting $a b$ holds and using (4.8), we have $a b b \rightarrow a b c \rightarrow a y b y c \rightarrow y=b$.

$$
(a, b) \cap(b, c)=\{b\} \text { implies } b \in(a, c) .
$$

3 The stronger pair of postulates, $(C)$ and their $(\beta)$, imply 42 of the 43 transitivities, all except $T_{10}$. 
Proof. We are to show that if $a x b \cdot b x c \rightarrow x=b$, then $a b c$ holds. This follows by choosing $x=(a, b, c)$.

$$
(a, b) \cap(b, c)=\{b\} \text { implies }(a, b) \cup(b, c)=(a, c) .
$$

Proof. Assume $a x b \cdot b x c \rightarrow x=b$. To show $a y c$ holds if and only if we have either $a y b$ or $b y c$. By (5.5), $a b c$ holds. The conclusion follows from (4.7) and (5.2).

$$
\text { If } d=(a, b, c) \text {, then }(a, b) \cap(b, c)=(b, d) .
$$

Proof. To show $b x d$ holds if and only if $a x b \cdot b x c$. Necessity of the condition is easily seen. Conversely, from (5.2),

$$
\begin{aligned}
& a x b \rightarrow(a x d b \text { or } a d x b) \rightarrow(a x d \text { or } d x b), \\
& b x c \rightarrow(b x d c \text { or } b d x c) \rightarrow(b x d \text { or } d x c) .
\end{aligned}
$$

If $b x d$ does not hold, we have from (4.8) the contradiction

$$
a d c \cdot a x d \cdot d x c \rightarrow a x d x c \rightarrow x=d .
$$

(5.8) Trees are characterized as sets $S$ satisfying Postulates B, C, and $\mathrm{D}_{1}$.

Proof. This follows from (2.4), (2.5), (2.6), (5.4), (5.6), and (5.7).

For later use we derive properties of the median. As a consequence of its definition, $(a, b, c)$ is invariant under cyclic permutations of $a, b$, and $c$. Hence from (4.3), we have the following.

(5.9) $(a, b, c)$ is invariant under all permutations of $a, b$, and $c$. As a consequence of (4.4) and (5.1) we have

$$
\begin{gathered}
(a, x, b)=x \text { if and only if } a x b \text { holds. Thus }(a, a, b)=a . \\
((x, a, b), c, x)=(x, a, c) \text { or }(x, b, c) .
\end{gathered}
$$

Proof. Let $y=(x, a, b)$ and $z=(y, c, x)$. By (4.7), xzy.xya $\rightarrow x z y a$ $\rightarrow x z a$. Similarly, we have $x z b$. Since $x z c$ holds, it remains to show that either $a z c$ or $b z c$ holds. If $y=z$, from (5.1) and (4.10) we have $a z b \cdot z c \rightarrow(a z c$ or $b z c)$. If $y \neq z$, we note $x z y a \rightarrow a y z$ and from (4.6) obtain $a y z \cdot y z c \rightarrow a y z c \rightarrow a z c$.

$$
((a, b, c),(a, b, d), e)=((c, d, e), a, b) .
$$

Proof. Let $x=(a, b, c), y=(a, b, d), r=(x, y, e), z=(c, d, e)$, and $s=(a, b, z)$. We are to prove $r=s$. From $a x b, a y b$, and (5.2), we have $a x y b$ or $a y x b$. Since these cases are handled similarly we assume:

(*) $a x y b$ holds. 
Case I. $x \neq r$ and $r \neq y$.

We have from $x r y$ that $x \neq y$. Using $(*)$, we have

$$
\begin{aligned}
b y x \cdot b x c & \rightarrow \text { byxc } \rightarrow c x y, \\
a x y \cdot a y d & \rightarrow \text { axyd } \rightarrow x y d, \\
c x y \cdot x y d \cdot x r y & \rightarrow \text { cxryd } \rightarrow c r d, \\
c x r y d \cdot e r y & \rightarrow \text { eryd } \rightarrow \text { erd },
\end{aligned}
$$

and

$$
\text { cxryd } \cdot \text { er } x \rightarrow \text { cxre } \rightarrow \text { cre. }
$$

But $c r d \cdot d r e \cdot e r c$ implies $r=(c, d, e)=z$. Moreover, from $\left(^{*}\right)$ and $x r y$, we have $a r b$. Hence from (5.10) we have $r=(a, r, b)=(a, z, b)=s$.

Case II. $x \neq r, r=y$.

We omit the proof since it is similar to the proof of Case III.

Case III. $x=r, r \neq y$.

We may assume $z \neq x$ for otherwise from $a x b$ we have $r=x=(a, b, x)$ $=s$. If $c z x$ holds we have $c z x \cdot c x b \rightarrow z x b$ and, similarly, $z x a$. Then $a x b \cdot b x z \cdot z x a$ imply $r=x=(a, b, z)=s$. It remains to show that to assume $c z x$ does not hold leads to a contradiction. Thus

$$
\begin{aligned}
c z d \cdot z x & \rightarrow(c z x \text { or } d z x) \rightarrow d z x, \\
c z e \cdot z x & \rightarrow(c z x \text { or } e z x) \rightarrow e z x, \\
e z x \cdot e x y & \rightarrow e z x y \rightarrow z x y,
\end{aligned}
$$

and

$$
d z x \cdot z x y \cdot z \neq x \rightarrow d z x y \rightarrow d x y .
$$

Finally, using $\left(^{*}\right)$, axy.ayd $\rightarrow x y d$. From $d x y \cdot x y d \cdot x \neq y \rightarrow d x y d$ we have the contradiction $x=d=y$.

Case IV. $x=r=y$.

To show $x=(a, b, c)=(a, b, d)$ and $z=(c, d, e)$ imply $x=(a, b, z)$. We note $c z d \cdot z x \rightarrow(c z x$ or $d z x)$. The cases are similarly treated. Assume, say, that $c z x$ holds. Then $c z x \cdot c x a \rightarrow z x a$ and $c z x \cdot c x b \rightarrow z x b$. Since $a x b$ holds, $r=x=(a, b, z)=s$.

6. Order betweenness. Consider a set $S$ satisfying Postulates $B, C$, and:

$\left(\mathrm{D}_{2}\right)$ For odd $n \geqq 3, a_{1} a_{2} \cdot a_{2} a_{3} \cdots a_{n-1} a_{n} \cdot a_{n} a_{1}$ implies either $a_{n-1} a_{n} a_{1}, a_{n} a_{1} a_{2}$, or $a_{i} a_{i+1} a_{i+2}$ for some $i, 1 \leqq i \leqq n-2$.

As a typical application of $\left(\mathrm{D}_{2}\right)$ we sketch the proof of transitivity $\mathrm{T}_{10}$ of Pitcher and Smiley [4]. 


$$
a b c \cdot a b d \cdot x b c \cdot a \neq b \cdot b \neq c \rightarrow x b d .
$$

Proof. The hypotheses imply $c x \cdot x b \cdot b d \cdot d a \cdot a c$. By $\left(\mathrm{D}_{2}\right)$, we have $c x b, x b d, b d a, d a c$, or $a c x$. If $c x b$ or $b d a$ holds, then $b$ equals $x$ or $d$ and $x b d$ holds. If $d a c$ or $a c x$ holds, we easily derive as a contradiction that $b$ equals $a$ or $c$.

In view of $(B),(4.3),(4.2),(4.6)$, and $\left(D_{2}\right)$, it is clear that the following theorem is a corollary of Altwegg's results [5, Sect. 2].

(6.2) Postulates $\mathrm{B}, \mathrm{C}$, and $\mathrm{D}_{2}$ characterize partially ordered sets to within dual orderings of their connected subsets. Here $a b c$ is equivalent to either $a \leqq b \leqq c$ or $c \leqq b \leqq a$.

It is possible to characterize a lattice in terms of betweenness by adding a fourth postulate to $(\mathrm{B}),(\mathrm{C})$, and $\left(\mathrm{D}_{2}\right)$. Then $a b c$ holds if and only if either $a+b=b c$ or $a b=b+c$. This is, of course, not the betweenness ordinarily called lattice betweenness. The latter, for distributive lattices, has $a b c$ equivalent to the condition $a c+b$ $=b(a+c)$.

7. Chain betweenness. A chain in the sense used here is often called a completely ordered set or a linearly ordered set. An extensive literature is devoted to discussion of chain betweenness.

Consider the postulate:

$\left(D_{3}\right)$ For $a, b$, and $c$ in $S$ either $a b c, b c a$, or $c a b$ holds.

(7.1) Chains are characterized by Postulates B, C, and $\mathrm{D}_{3}$ or by Postulates $\mathrm{B}, \mathrm{C}, \mathrm{D}_{1}$, and $\mathrm{D}_{2}$.

Proof. The first statement follows from a result of Altwegg [5, Sect. 4] and from $\left(D_{3}\right),(4.3),(B),(4.6)$, and (4.7). Since a chain is clearly both a tree and a partially ordered set, it remains to be proved only that $(B),(C),\left(D_{1}\right)$, and $\left(D_{2}\right)$ imply $\left(D_{3}\right)$. This follows from (5.1) and $\left(\mathrm{D}_{2}\right)$, since $a b \cdot b c \cdot c a$ implies $a b c, b c a$, or $c a b$.

8. Medians. Some of the work in this section is similar to previous work (see, for example, [3, p. 137]). It has not, however, been previously shown that the results can be made independent of Postulate $\mathrm{O}_{2}$ below.

Consider a set $S$ closed under a ternary operation $(a, b, c)$, called the median of $a, b$, and $c$, satisfying the following postulates:

(M) $(a, a, b)=a$.

(N) $((a, b, c),(a, b, d), e)=((c, d, e), a, b)$.

The following theorem is proved by setting $c=d$ in $(\mathrm{N})$ and applying $(\mathrm{M})$. We have (8.2) as a corollary. 


$$
\begin{aligned}
& (a, b, c)=(c, a, b) . \\
& (b, a, a)=(a, b, a)=(a, a, b)=a .
\end{aligned}
$$

The next theorem is used freely in the work to follow.

(8.3) The median $(a, b, c)$ is invariant under permutations of $a, b$, and $c$.

Proof. In view of (8.1), it is sufficient to show $(a, b, c)=(b, a, c)$. This follows from the equalities

$$
\begin{aligned}
(a, b, c) & =((b, a, a),(b, a, b), c)=((a, b, c), b, a) \\
& =((a, b, c),(a, b, b), a)=((c, b, a), a, b) \\
& =((b, a, c), a, b)=((a, b, b),(a, b, a), c)=(b, a, c) .
\end{aligned}
$$

The next theorem is a direct consequence of $(\mathrm{N})$ and (8.2).

$$
\begin{aligned}
((a, b, c), a, b) & =(a, b, c) . \\
((a, x, b),(b, x, c),(c, x, a)) & =((x, a, c), b, x) .
\end{aligned}
$$

Proof. By $(\mathrm{N})$ and (8.4) both expressions equal $((a, c,(c, x, a)), b, x)$. As a corollary we have

(8.6) $\quad((x, a, c), b, x)$ is invariant under permutations of $a, b$, and c.

$$
((a, b, c),(a, b, d),(a, b, e))=((c, d, e), a, b) .
$$

Proof. Using $(\mathrm{N})$ and (8.6), we have

$$
\begin{aligned}
((a, b, c),(a, b, d),(a, b, e)) \\
\quad=(((a, b, d),(a, b, e), a),((a, b, d),(a, b, e), b), c) \\
\quad=(((d, e, a), a, b),((d, e, b), a, b), c) \\
\quad=(((d, b, a), a, e),((d, a, b), e, b), c) \\
\quad=((a, b, c),(d, b, a), e)=((c, d, e), a, b) .
\end{aligned}
$$

Definition. We say $x$ is between $a$ and $b$ and write $a x b$ if and only if $x=(a, x, b)$. Extensions of this notation such as $a b c d$ are made as in $\$ 4$.

The next two theorems are immediate.

$$
\begin{gathered}
a b c \rightarrow c b a \\
a b c \cdot a c b \leftrightarrow b=c .
\end{gathered}
$$

Hence $a a b$ holds and $a b a \leftrightarrow a=b$. 
$a b c \cdot b c d \cdot a d e \rightarrow b c e$.

Proof. We have $(b, c, e)=((a, b, c),(b, c, d), e)=((a, d, e), b, c)$ $=(d, b, c)=c$.

$$
a b c \cdot a b d \cdot c e d \rightarrow a b e .
$$

Proof. We have $(a, b, e)=(a, b,(c, d, e))=((a, b, c),(a, b, d), e)$ $=(b, b, e)=b$.

The implications just established are found in [4] under the labels $\alpha, \beta, \mathrm{T}_{4}$, and $\mathrm{T}_{7}$. We have from $\S 6$ of that paper the following as consequence.

$$
a b c \cdot a c d \rightarrow a b c d
$$

(8.13) Given $a, b$, and $c$, there is a unique element $x$ such that $a x b \cdot b x c \cdot c x a$.

Proof. From (8.4), $(a, b, c)$ satisfies these betweenness relations. If the relations hold for both $x$ and $y$ then $x=(x, x, y)=((a, x, b)$, $(a, x, c), y)=((b, c, y), a, x)=(y, a, x)$ and, similarly, $y=(y, a, x)$.

If we define $(a, b)$ as the set of all $x$ such that $x=(a, x, b)$ it is not difficult to prove the following.

\section{Postulates $\mathrm{M}$ and $\mathrm{N}$ imply Postulates $\mathrm{S}$ and $\mathrm{T}$.}

9. Tree medians. Consider Postulates $\mathrm{M}, \mathrm{N}$, and:

$\left(\mathrm{O}_{1}\right)((x, a, b), c, x)=(x, a, c)$ or $(x, b, c)$.

It is clear from (8.6) and (8.7) that this postulate can be restated as follows. The elements $(a, x, b),(b, x, c)$, and $(c, x, a)$ are not distinct.

We wish to show these postulates characterize trees. By (5.10), (5.11), and (5.12), Postulates $B, C$, and $D_{1}$ imply Postulates $M, N$, and $\mathrm{O}_{1}$. Conversely, by (8.9) and (8.13), $(\mathrm{M}),(\mathrm{N})$, and $\left(\mathrm{O}_{1}\right)$ imply (B) and $\left(\mathrm{D}_{1}\right)$. To show (C) also holds let $b=(a, b, c), d=(b, d, e)$, and $b \neq(c, b, d)$. By (8.2), $b \neq d$. By $\left(\mathrm{O}_{1}\right), b=(b, d, b)=((b, c, a), d, b)$ $=(b, a, d)$. Hence $b=((b, a, d), e, b)=(b, a, e)$ or $(b, d, e)$. To assume the latter gives the contradiction $b=d$. Hence $b=(b, a, e)$ and $e b a$ holds. By (5.8), the following holds.

(9.1) Postulates $\mathrm{M}, \mathrm{N}$, and $\mathrm{O}_{1}$ characterize trees.

10. Lattice medians. Consider Postulates $M, N$, and:

$\left(\mathrm{O}_{2}\right)$ There are elements 0 and $I$ in $S$ such that for all $a$ in $S$, $a=(0, a, I)$.

In view of (8.3), the following is a restatement of a known theorem $[3$, p. 137]. 
(10.1) Distributive lattices with bounds 0 and $I$ are characterized by Postulates $\mathrm{M}, \mathrm{N}$, and $\mathrm{O}_{2}$. This is achieved by use of the relations $a b=(a, 0, b), a+b=(a, I, b)$, and $(a, b, c)=a b+b c+c a$.

It is possible to characterize these lattices by means of only two postulates based on medians (see [6]).

11. Chain medians. Results in this section are similar to those in $\$ 7$. We consider the postulate

$\left(\mathrm{O}_{3}\right)$ If $(a, b, c) \neq a$ or $c$, then for all $x$ either $(a, x, b)$ or $(b, x, c)=b$.

A simple four element example shows that the following cannot replace $\left(\mathrm{O}_{3}\right)$ in (11.2). Postulates $\mathrm{M}, \mathrm{N}$, and $\mathrm{O}_{3}$ imply

$$
(a, b, c)=a, b \text {, or } c .
$$

Proof. Let $x$ in $\left(\mathrm{O}_{3}\right)$ be $(a, b, c)$ and apply (8.4). (11.2) Postulates $\mathrm{M}, \mathrm{N}$, and $\mathrm{O}_{3}$ characterize chains.

Proof. Assume $(\mathrm{M}),(\mathrm{N})$, and $\left(\mathrm{O}_{3}\right)$. Postulates $\mathrm{B}$ and $\mathrm{D}_{3}$ follow by (8.9) and (11.1). By (7.1), we have a chain if Postulate $C$ holds. Assume $a b c \cdot b d e$. If $e b a$, (C) holds. If $b e a$, we have in turn $b d e a$, $c b d e a, c b d$, and (C) holds. But if neither $e b a$ or $b e a$ holds, we have by $\left(\mathrm{O}_{3}\right)$ either $b a c$ or $c a e$. In the first case, $b a c \cdot a b c \rightarrow a=b \rightarrow e b a$, a contradiction. In the second case, we have in turn $e a b c, e b c, e d b c$, and $d b c$. Again, (C) holds. Conversely, since a chain is a tree it satisfies Postulates $\mathrm{M}$ and $\mathrm{N}$. It remains to show $\left(\mathrm{O}_{3}\right)$ holds. Assume $(a, b, c) \neq a$ or $c$. By $\left(\mathrm{D}_{3}\right)$ we have $a b c$. If $a b x,\left(\mathrm{O}_{3}\right)$ follows. If not, $a \neq b$ and, from $\left(\mathrm{D}_{3}\right)$, either $b x a$ or $b a x$ holds. In the first case, $a b c \cdot b x a \rightarrow(c b x$ or $a b a) \rightarrow c b x$. In the second case, $c b a \cdot b a x \cdot a \neq b \rightarrow c b a x$ $\rightarrow c b x$.

It is clear from the following that Postulates $M, N, O_{1}$, and $\mathrm{O}_{2}$ characterize bounded chains.

(11.3) Postulates $\mathrm{M}, \mathrm{N}, \mathrm{O}_{1}$, and $\mathrm{O}_{2}$ imply Postulate $\mathrm{O}_{3}$.

Proof. Since $(a, 0, b)=((0, a, b), I, 0)=(0, a, I)$ or $(0, b, I)$, we have, say, $(a, 0, b)=a$. Then $(a, b, c)=((0, b, a),(0, b, b), c)$ $=((a, b, c), 0, b)=(b, a, 0)$ or $(b, c, 0)$. Hence $(a, b, c)=a, b$, or $c$. If $(a, b, c)=b, b=(b, x, b)=((b, a, c), x, b)=(b, a, x)$ or $(b, c, x)$ and $\left(\mathrm{O}_{3}\right)$ is established.

12. Median latticoids. It is interesting that trees and bounded distributive lattices have an algebra with many properties in common. Assume Postulates $\mathrm{M}$ and $\mathrm{N}$ hold for a set of elements $S$. Let 0 and $I$ be elements arbitrarily chosen from $S$. Define $a+b$ as $(a, I, b)$ and $a b$ as $(a, 0, b) . S$ is closed under these operations and 
properties $\mathrm{V}$ and $\mathrm{W}$ are immediate. Properties $\mathrm{X}$ and $\mathrm{Y}$ follow respectively from (8.6) and (N).

(V) The idempotent laws hold.

(W) The commutative laws hold.

(X) The associative laws hold.

(Y) The distributive laws hold and hence $a+a b=a(a+b)$.

(Z) Elements 0 and $I$ have the properties that, for all $a$ and $b$ in $S, a 0=0, a+I=I$, and $a+0=I a=I(a+a b)$.

Proof. All properties except the last are immediate. The last follows from $(I, 0,(a, I,(a, 0, b)))=(I, 0,(a, b,(a, 0, I)))=((I$, $0, a), b,(I, 0,(a, 0, I)))=((I, 0, a), b,(a, 0, I))=(I, 0, a)$.

\section{BiBLIOGRAPHY}

1. W. D. Duthie, Segments of ordered sets, Trans. Amer. Math. Soc. vol. 51 (1942) pp. 1-14.

2. Dénes König, Theorie der endlichen and unendlichen Graphen, Leipzig, 1936.

3. Garrett Birkhoff, Lattice theory, Amer. Math. Soc. Colloquium Publications, vol. 25, rev. ed., 1948.

4. E. Pitcher and M. F. Smiley, Transitivities of betweenness, Trans. Amer. Math. Soc. vol. 52 (1942) pp. 95-114.

5. Martin Altwegg, Zur Axiomatik der teilweise geordneten Mengen, Comment. Math. Helv. vol. 24 (1950) pp. 149-155.

6. Marlow Sholander, Postulates for distributive lattices, Canadian Journal of Mathematics vol. 3 (1951) pp. 28-30.

WASHINGTON UNIVERSITY 